\title{
„Die kleinen Taschen-Sonografiegeräte gehören schon fast - wie früher das Stethoskop - in die Kitteltasche des Aufnahme- und Stationsarztes“
}

\author{
Kongresspräsidenten des Dreiländertreffens Prof. Dr. med. Markus Hahn (Tübingen) und \\ Dr. Hans-Peter Weskott (Hannover) im Gespräch
}

Das Ultraschallverfahren hat in den vergangenen Jahren eine rasante Entwicklung hingelegt. Beim Dreiländertreffen der Fachgesellschaften DEGUM, ÖGUM und SGUM in Leipzig vom 16. bis 19. Oktober werden unter dem Motto „Mit UItraschall die Gegenwart und Zukunft gestalten" die neuesten wissenschaftlichen Erkenntnisse präsentiert sowie Fortbildung in Theorie und Praxis angeboten. „Wir sehen uns als Fachgesellschaften in der Pflicht, die Ärzte aller Fachrichtungen auszubilden und gleichzeitig die Forschung zu fördern“, sagen die Kongresspräsidenten Prof. Dr. med. Markus Hahn und Dr. Hans-Peter Weskott. Die Sonografie gehört zu den wichtigsten und am häufigsten eingesetzten bildgebenden Verfahren in der Medizin.

Herr Professor Hahn, Herr Dr. Weskott, das war doch bestimmt ein Schock, als Sie vom Brand in der Rheingoldhalle am 17. Mai erfahren haben?

Dr. Weskott: So etwas ist in der 43-jährigen Geschichte des Dreiländertreffens noch nicht passiert. Umso dankbarer sind wir, dass der Kongressveranstalter so schnell reagieren konnte und wir mit dem Congress Center in Leipzig einen so hervorragenden, bewährten und gut erreichbaren Austragungsort gefunden haben.

Es kann in Leipzig alles wie geplant stattfinden?

Prof. Hahn: Wir wollen als DEGUM einen Kongress in höchster Qualität anbieten. Dafür waren die Räumlichkeiten in Mainz leider nicht mehr nutzbar. In Leipzig können

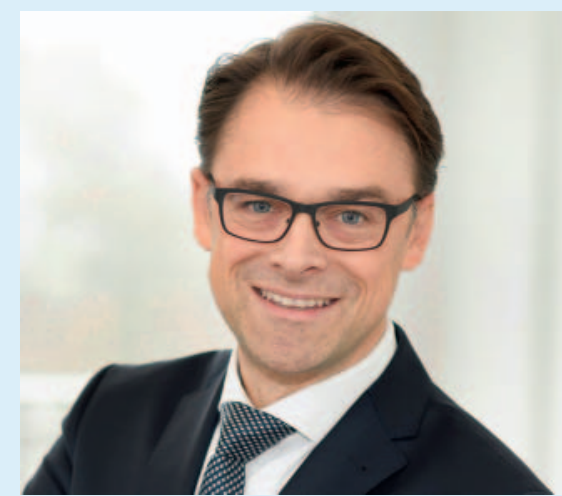

Kongresspräsident Prof. Markus Hahn. Quelle: Universitätsklinikum Tübingen, Fotografin Britt Moulien

wir das gesamte Programm, alle Vorträge, Live-Präsentationen, Anwenderseminare und die Industrieausstellung ohne Abstriche wie geplant anbieten. Natürlich bringt es einen enormen Mehraufwand mit sich, jetzt noch mal alle Planungen an die neuen Räumlichkeiten anzupassen. Aber es wird definitiv gelingen.

Die DEGUM ist mit 11000 Mitgliedern die größte interdisziplinäre medizinische Fachgesellschaft in Deutschland. Wie schafft man es, die vielen Fachdisziplinen aus drei Ländern unter einen Hut zu bringen?

Dr. Weskott: Das Interessante und Besondere bei unserem Kongress ist die Kommunikation und der Austausch zwischen den verschiedenen Fachdisziplinen. Man lernt voneinander, bekommt Input, welche Techniken wie und wo eingesetzt werden. Das ist ein Riesengewinn.

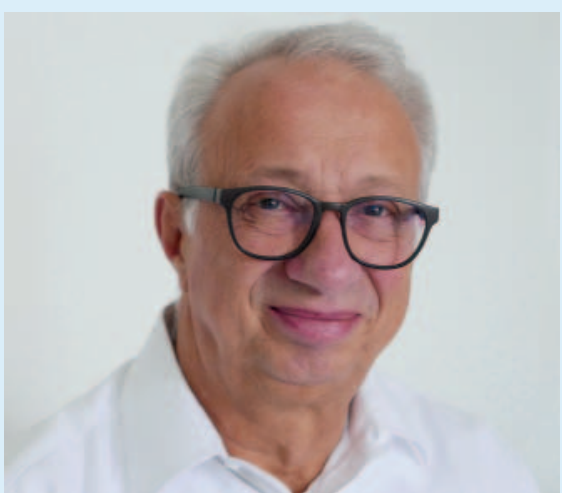

Kongresspräsident Dr. Hans-Peter Weskott. Quelle: Dr. Hans-Peter Weskott, Hannover

Prof. Hahn: Sonografie ist das, was früher das Stethoskop war - sogar noch viel mehr. Wir können heute mit dem Ultraschall hochauflösend die anatomischen Verhältnisse in allen Organen und Gefäßarealen darstellen; somit wird das Verfahren von allen Fachdisziplinen in allen Bereichen eingesetzt. Umso wichtiger ist es, dass die Mediziner die Ultraschalltechnik gut beherrschen. Als Fachgesellschaft sehen wir uns deshalb in der Pflicht, die Ärzte aller Fachrichtungen auszubilden und gleichzeitig die Forschung zu fördern.

Das diesjährige DLT steht unter dem Motto „Mit Ultraschall die Gegenwart und Zukunft gestalten“. Was sind die Ihrer Meinung nach bedeutsamsten Errungenschaften des Ultraschalls von heute und morgen?

Dr. Weskott: Wesentliche Meilensteine in der Diagnostik war die Einführung der kontrastverstärkten Sonografie sowie der Elas- 
tografie, wodurch wir physiologische und pathophysiologische Vorgänge besser verstehen und die Diagnostik immer weiter optimieren können. Der jährliche diagnostische Zugewinn ist weiterhin beträchtlich, erfordert aber auch ständiges „Dazulernen“. Dazu haben wir am Mittwoch die Anwenderseminare im Programm, in denen ganztägig schwerpunktmäßig Ultraschallthemen vermittelt werden. Von Donnerstag bis Freitag bieten wir Refresher-Kurse an, am Samstag auch hausärztliche Themen. Die wissenschaftlichen Beiträge aus allen medizinischen Disziplinen finden von Donnerstag bis Freitag statt. Es werden aber auch Themen aufgegriffen, die für jeden Hausarzt und sein Patientenmanagement im Praxisalltag wichtig sind.

Prof. Hahn: Die Sonografie ist längst auch in den operativen Fächern angekommen. So ist es heute selbstverständlich, das Ultraschallgerät mit in den OP zu nehmen oder ein bereits dort installiertes System zu nutzen. Das ultraschallunterstützte Operieren ermöglicht Regionen einzusehen, die visuell auch mit der Lupenbrille nicht sichtbar sind. So werden zum Beispiel Karzinomoperationen sehr viel exakter.

\section{Der Ultraschall wird also auch zunehmend in der Krebsbehandlung eingesetzt?}

Prof. Hahn: Auf ganz unterschiedlichen Ebenen sogar. Wir haben mit dem hochintensiven fokussierten Ultraschall - HIFU die Möglichkeit, kleinere Tumoren millimetergenau zu zerstören. Dabei werden die Schallwellen so stark gebündelt, dass durch den thermischen Effekt Temperaturen von bis zu 80 Grad Celsius auf ein nur wenige Millimeter großes Gebiet entstehen. Hier ersetzt der Ultraschall im Prinzip das Skalpell - ohne die Gefahr von Blutungen oder Tumorverschleppung.

Dr. Weskott: In der Onkologie wird aber auch zunehmend die Kontrastmittelsonografie angewendet. So können wir hiermit zu einem sehr frühen Zeitpunkt sehen, ob beispielsweise eine Chemo-, Immun- oder Strahlentherapie erfolgreich ist oder ohne Wirkung auf die Tumorentwicklung bleibt, was im CT erst viel später sichtbar wird.
Prof. Hahn: Sonografie kann auch interventionell eingesetzt werden. So haben wir schon seit langem ultraschallgesteuerte Biopsie-Nadeln zur Verfügung, mit denen wir Gewebe entnehmen können, ohne operieren zu müssen. Das kann zum Beispiel beim Mammografie-Screening vorteilhaft sein. Bei Verdacht entnehmen wir somit sehr exakt und damit nur ganz wenig Gewebe, das uns im Anschluss jede Information liefert - auch auf welche Medikamente der Tumor reagiert.

\section{Der Ultraschall ist ein wirkliches Multita- lent.}

Dr. Weskott: Definitiv. Und absolut universell und zukunftsweisend. Die am schnellsten wachsende Mitgliederzahl in der DEGUM finden wir bei den Anästhesisten. Die Möglichkeit der sonografischen Darstellung von Nerven und Muskeln ermöglicht gezielte lokale Therapien sowie von präoperativen Nervenblockaden. Auch die Rheumatologen und Neurologen vertrauen immer öfter den Vorteilen der sonografischen Techniken. Der hochaufgelöste Ultraschall im Operationssaal macht ein ganz anderes Operieren möglich als das früher der Fall war. Und auch auf dem Gebiet der Notfallmedizin ermöglicht uns die technische Entwicklung immer kleinerer Geräte und Sonden eine schnellere und bessere Diagnostik am Unfallort.

Und die Entwicklung geht ja noch weiter: Künstliche Intelligenz, molecular imaging - sind das einige Highlights des Dreiländertreffens?

Dr. Weskott: Wir werden neue Einsatzgebiete für die KI sowie bei der Entwicklung neuer Kontrastmittel zur Diskussion stellen. Daneben ist es uns sehr wichtig, den Ultraschall in der Diagnostik und Therapie ganz umfassend vorzustellen - natürlich auch neue zukunftsweisende High-end-Techniken, über die man sich unter anderem auch auf der Industrieausstellung informieren kann.
Prof. Hahn: Wir wollen die Qualität des Untersuchungsverfahrens aufzeigen. Erfahrene Ultraschall-Untersucher, Studierende und Berufsanfänger können sich beim diesjährigen Dreiländertreffen wieder auf den neuesten Stand bringen lassen.

Apropos Studierende: Wenn der Ultraschall das neue Stethoskop sein soll, müssten Studenten doch sehr früh die Techniken erlernen. Wie geht die DEGUM damit um?

Prof. Hahn: Die DEGUM bietet Studenten eine Plattform, wo sie von Experten lernen können. So haben wir spezielle Curricula für Studenten entwickelt. Auf dem Kongress wird es allein 6 Sessions nur für Studierende geben, bei denen alle Organ- und Gefäßsysteme auf dem Programm stehen. Außerdem ist ein ganzer Tag geplant, bei dem die Studierenden an Probanden schallen können und dabei von Experten angeleitet werden. Und natürlich stellen die kleinen Ultraschallgeräte, die man in der Kitteltasche überall hin mitnehmen kann, nur einen kleinen Teil unseres Programms dar: High-end-Ultraschalltechniken von außen und innen eingesetzt - wie Endosonografie und der intraoperative Ultraschall - werden einen festen Platz im Programm einnehmen.

Das Dreiländertreffen ist seit jeher auch eine Plattform für gesundheitspolitische Themen. Welche politischen Entwicklungen treiben Sie um?

Prof. Hahn: Das deutsche Gesundheitssystem ist eines der besten auf der Welt. Dennoch hat jedes System seinen Preis. Und so wollen wir uns mit der Frage beschäftigen: Ist es für uns denkbar, den Ultraschall in bestimmten Bereichen an nichtmedizinische Anwender abzugeben? Wollen wir das? Wir werden das diskutieren.

Dr. Weskott: Und wir wollen auch das Thema der Sicherheit des Ultraschalls noch einmal aufgreifen und mit Experten der DEGUM und EFSUMB dieses Thema diskutieren, um entstandene Irritationen auszuräumen.

Das Gespräch führte Katharina Weber 